\title{
The thiJ locus and its relation to phosphorylation of hydroxymethylpyrimidine in Escherichia coli
}

\author{
Tomoko Mizote, ${ }^{1}$ Masataka Tsuda, ${ }^{2}$ Teruko Nakazawa ${ }^{2}$ \\ and Hideo Nakayama' + \\ Author for correspondence: Hideo Nakayama. Tel: +81 83658 5360. Fax: + 81836585362.
}

1 Department of Food and Nutrition, Yamaguchi Prefectural University, 3-2-1 Sakurabatake, Yamaguchi 753, Japan

2 Department of Microbiology, Yamaguchi University School of Medicine, Ube, Yamaguchi 755 , Japan

\begin{abstract}
Extracts from Escherichia coli K-12 contained two distinct enzymes capable of catalysing the phosphorylation of hydroxymethylpyrimidine (HMP) to HMP monophosphate: pyridoxine kinase (EC 2.7.1.35) and an enzyme that has not previously been genetically analysed, HMP kinase (EC 2.7.1.49). Two distinct genes, $p d x L$ and this, specify the activities of the former and latter enzymes, respectively. The inactivation of both genes by independent mutations in the same cell resulted in the complete loss of HMP kinase activity. Experiments with a series of strains that carry mutations in thiC, thiC pdxB, thiC pdxB pdxL and thiC $p d x B$ pdxL thiJ revealed that the ability of the double mutant (pdxL thif) to utilize HMP in thiamin pyrophosphate biosynthesis was restored by introducing the wild-type allele corresponding to the this mutation. The thiJ locus was mapped on the chromosome near the thiD and thiM loci, which govern the activities of phosphomethylpyrimidine kinase (EC 2.7.4.7) and hydroxyethylthiazole kinase (EC 2.7.1.50), respectively.
\end{abstract}

Keywords: Escherichia coli, thiamin biosynthesis, thiJ locus, hydroxymethylpyrimidine

\section{INTRODUCTION}

In Escherichia coli five enzymes are involved in the biosynthesis of thiamin pyrophosphate (thiamin-PP) from 2-methyl-4-amino-5-hydroxymethylpyrimidine (hydroxymethylpyrimidine, HMP) and 4-methyl-5-( $\beta$-hydroxyethyl)thiazole (hydroxyethylthiazole, HET) via the intermediate formation of thiamin monophosphate (thiamin-P) (Nakayama \& Hayashi, 1972a). Another enzyme catalyses the formation of thiamin-P from thiamin (Nakayama \& Hayashi, 1972b). The genes specifying five of these six enzymes, thiB, thiD, thiK, thiL and thiM, are distributed on the chromosome in four distinct regions (Kawasaki et al. 1968; Mizote \& Nakayama, 1989b) (Fig. 1). Among these, thiD and thiM, which code for phosphomethylpyrimidine (hydroxymethylpyrimidine monophosphate, HMP-P) kinase (EC 2.7.4.7) and HET kinase (EC 2.7.1.50) activities, respectively, are located

t Present address: Department of Internal Medicine, Sea-Side Hospital, Maruo, Higashikiwa, Ube 755-02, Japan.

Abbreviations: HMP (hydroxymethylpyrimidine), 2-methyl-4-amino-5hydroxymethylpyrimidine; HMP-P, HMP monophosphate; HMP-PP, HMP pyrophosphate; HET (hydroxyethylthiazole), 4-methyl-5-( $\beta$-hydroxyethyl)thiazole; thiamin-P, thiamin monophosphate; thiamin-PP, thiamin pyrophosphate; POL, pyridoxol; POL-P, pyridoxol 5-phosphate; PAL, pyridoxal PAL-P, pyridoxal 5-phosphate; PAM, pyridoxamine. close together on the chromosome at about $46 \mathrm{~min}$ (Mizote \& Nakayama, 1989b). However, genetic analysis of HMP kinase (EC 2.7.1.49), which catalyses the phosphorylation of HMP to HMP-P, has not been possible because strains deficient in this enzyme have never been reported. We previously characterized an

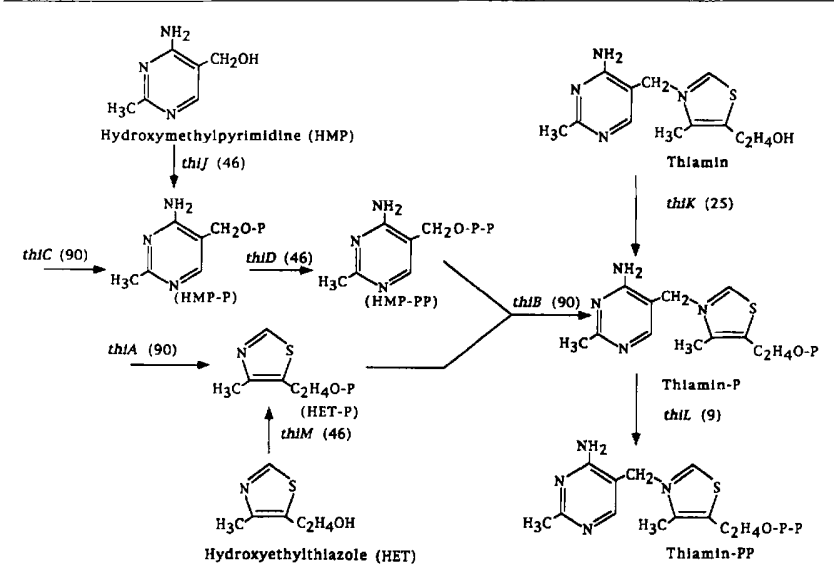

Fig. 1. Biosynthetic pathway for thiamin-PP from HMP and HET in $E$. coli. Parentheses indicate approximate map positions ( $\mathrm{min}$ ) of each of the genes. 
HMP-phosphorylating enzyme purified from E. coli K-12 cells grown in minimal medium and showed that the enzyme phosphorylates each of the pyridoxine substrates, pyridoxol (POL), pyridoxal (PAL) and pyridoxamine (PAM) as well as HMP and that the phosphorylation of HMP is competitively inhibited by POL and vice versa. This led to the conclusion that HMP is phosphorylated by the enzyme previously designated pyridoxine kinase (PN/PL/PM kinase; pyridoxal kinase; EC 2.7.1.35), (Mizote \& Nakayama, 1989a).

Subsequently, we succeeded in isolating strains that showed a reduced kinase activity for both HMP and POL by a second mutation in a strain already carrying the $p d x B$ mutation. For the second mutated gene we tentatively gave the designation thi $N$ (Nakayama, 1990). However, our present investigation has revealed that pyridoxine kinase is lacking in this class of strains, even though extracts from the strain possess lower HMP kinase activity than the parent strain. This reduction in activity has no effect on the utilization of HMP for thiamin-PP biosynthesis in the living cell and led us to change the designation of this gene from thiN to $p d x L$. Additional evidence in support of this redesignation was obtained from experiments with a strain totally defective in HMP kinase. The locus of the new mutation, for which the symbol thij will be used here, was situated in a region near the thiD and thiM loci.

\section{METHODS}

E. coli strains. The bacterial strains constructed for this study are listed in Table 1. The principal strains used in the experiments are all derived from a polyauxotrophic strain,
JC1552. The biosynthesis of thiamin-PP in this strain from HMP and HET has been described previously (Imamura \& Nakayama, 1982).

Media and growth conditions. Minimal medium 56 as modified by Low (1973) was used for the growth experiments including the selection for the desired strains. Mutants reported here were produced by treatment of parent cells with $\mathrm{N}$-methyl- $\mathrm{N}^{\prime}$-nitro$\mathrm{N}$-nitrosoguanidine (N'TG) by the method reported by Adelberg et al.(1965). In some experiments ethylmethane sulfonate (EMS) was used, following the method of Osborn et al. (1967). Mating and strain constructions were performed by procedures essentially as described by Low (1973) and Miller (1972). Phage P1 kc-mediated transductions were performed according to Lennox (1955) and Taylor \& Trotter (1967). Luria broth was used in transduction experiments, and Luria broth without glucose was used in conjugation experiments.

Enzyme assay. Enzyme activity with crude extracts and with whole cells were measured as described previously (Nakayama \& Hayashi, 1972b). The amounts of HMP-P and hydroxymethylpyrimidine pyrophosphate (HMP-PP) formed were estimated by microbiological assay with paper bioautography using E. coli strain 70-17. Detailed assay conditions with this strain have been described previously (Nakayama \& Hayashi, 1979). The amount of phosphate esters of the pyridoxine substrates formed was assayed with E. coli strain $\mathrm{H} 5-619$, a strain auxotrophic for pyridoxine, by essentially the same procedure as that for HMP-P.

Chemicals. HMP, 2-methylthio-4-amino-5-hydroxymethylpyrimidine (methylthiohydroxymethylpyrimidine) and 2methyl-4-amino-5-bromomethylpyrimidine dihydrobromide (bromomethylpyrimidine) were gifts from Takeda Chemical Industries, Osaka, Japan. HMP-P and HMP-PP were prepared from bromomethylpyrimidine according to the method of Lewin \& Brown (1961). POL, PAL, PAM, 4-deoxypyridoxine and pyridoxal 5-phosphate (PAL-P) were purchased from

Table 1. E. coli strains

\begin{tabular}{|c|c|c|c|}
\hline Strains & Relevant genotype* & $\begin{array}{c}\text { Relevant } \\
\text { phenotype } \dagger\end{array}$ & Source \\
\hline JC1552 & argG6 metB1 bis-1 leu-6 trp-31 rpsL104 & & CGSC $\$$ \\
\hline SN372 & $\mathrm{JC} 1552$ thiC21 & $\mathrm{Hmp}^{-}$ & Isolated from JC1552 by mutagenesis \\
\hline SN372L & SN372 leu $u^{+}$ & $\mathrm{Hmp}^{-}$ & $\mathrm{SN} 372 \times \mathrm{BW} 113$ \\
\hline SN372L-3 & SN372L $p d x B 20$ & $\mathrm{Hmp}^{-} \mathrm{Pol}^{-}$ & Isolated from SN372L by mutagenesis \\
\hline SN372L-3-9 & SN $372 \mathrm{~L}-3 p d x \mathrm{~L} 2$ & $\mathrm{Hmp}^{-} \mathrm{Pal}^{-}$ & Isolated from SN372L-3 by mutagenesis \\
\hline SN372L-3-9-10 & SN372L-3-9 thiJ1 & $\mathrm{Thi}^{-} \mathrm{Pal}^{-}$ & Isolated from SN372L-3-9 by mutagensis \\
\hline M-3-9-6 & SN372L-3-9-10 tbij ${ }^{+}$tbiM1 & $\mathrm{Hmp}^{-} \mathrm{Pal}^{-}$ & SN372L-3-9-10 × P1kc (SN164L-1) \\
\hline K3-9-101 & SN372L-3-9-10 $p d x L^{+}$nupC3146:: $\operatorname{Tn} 10 k a n$ & $\mathrm{Thi}^{-} \mathrm{Pol}^{-}$ & SN372L-3-9-10 × P1kc (CAG18565) \\
\hline SN372L-3-T1 & SN372L-3 pdxL2 nupC3146:: Tn10kan & $\mathrm{Hmp}^{-} \mathrm{Pol}^{-}$ & SN372L-3 × P1kc (SNK372L-3-9-10) \\
\hline SN164L-1 & JC1552 leu ${ }^{+}$thiA7 thiM1 & $\mathrm{Thi}^{-}$ & Mizote \& Nakayama (1989b) \\
\hline AB2557 & purF1 aro $C 4 d s d A 1$ & & CGSC $\ddagger$ \\
\hline $\mathrm{AB} 2557 \mathrm{~F}$ & $\mathrm{AB} 2557$ purF $^{+} p d x \mathrm{~B} 20$ & $\mathrm{Pol}^{-}$ & $\mathrm{AB} 2557 \times \mathrm{P} 1 k c(\mathrm{SN} 372 \mathrm{~L}-3)$ \\
\hline CAG18565 & nupC3146:: Tn10kan & & NIGS \\
\hline
\end{tabular}

* Genetic nomenclature is as given by Bachmann (1983).

$\dagger \mathrm{Hmp}^{-}$, strains requiring either thiamin or HMP for growth; Thi ${ }^{-}$, strains requiring thiamin for growth, and incapable of growth on either HMP or HET; $\mathrm{Pol}^{-}$, strains requiring POL or PAL at concentrations above $0 \cdot 1 \mu \mathrm{M}$ for maximum growth; Pal ${ }^{-}$, strains capable of growth on PAL to the same extent as $\mathrm{Pol}^{-}$strains, but incapable of growth on POL at concentrations below $0 \cdot 1 \mu \mathrm{M}$ and requiring POL at concentrations above $10 \mu \mathrm{M}$ for maximum growth.

$\ddagger$ CGSC, Coli Genetic Stock Center, Department of Biology 255 OML, Yale University, New Haven, CT, USA.

$\S$ NIG, National Institute of Genetics, Department of Microbiology, Mishima, Shizuoka, Japan. 
Sigma. Pyridoxol 5-phosphate (POL-P) was a gift from Dr A. Iwashima.

\section{RESULTS AND DISCUSSION}

\section{Isolation and characterization of a pdxL mutant}

Our previous study (Nakayama, 1990) showed that strains with reduced HMP kinase activity can be isolated from a $p d x B$ strain by selection for deoxypyridoxine resistance. All of the resistant strains isolated showed the same growth characteristics $\left(\mathrm{Pol}^{-}\right)$as described in the footnote of Table 1. To determine whether or not the strains of this class can utilize HMP for thiamin-PP biosynthesis, a thiC strain which requires HMP for growth was initially produced, followed by the isolation of a series of strains with additional mutations in the loci of $p d x B, p d x L$ and thij.

The thiC (Ts) $p d x B$ (Ts) strain (SN372L-3) was mutagenized with EMS and cultured in minimal medium containing $20 \mathrm{nM}$ thiamin and $0.1 \mu \mathrm{M}$ PAL at $37^{\circ} \mathrm{C}$ for $24 \mathrm{~h}$. The culture was washed with saline and a portion was mixed in minimal agar to yield approximately $10^{9}$ cells per plate. A paper disk containing $10 \mathrm{nmol}$ deoxypyridoxine was placed on the centre of the plate and the plate was incubated at $25^{\circ} \mathrm{C}$ for $72 \mathrm{~h}$. Resistant colonies growing near the paper were isolated at random and purified. Mutants that could grow on $0 \cdot 2 \mu \mathrm{M}$ PAL but not on $0.2 \mu \mathrm{M}$ POL at $37^{\circ} \mathrm{C}$ were selected using medium containing thiamin by the replica-plating method of Lederberg \& Lederberg (1952). It has been shown that POL-P oxidase can catalyse the conversion from POL-P into PAL-P in E. coli (Turner \& Happold, 1961; Henderson, 1965) and that the $p d x H$ mutation, which is responsible for the loss of the oxidase activity and for the growth requirement of $\mathrm{PAL}$, is located at $36 \mathrm{~min}$ on the genetic map (Shimizu \& Dempsey, 1976; Lam \& Winkler, 1992). Our $p d x L$ mutants differ from $p d x H$ mutants in their enzymic deficiencies, POL requirements and genetic map positions.

\section{Location of $p d x L$}

Preliminary matings placed the site of the $p d x L$ gene between $\sup N$ and $d s d A$ on the chromosome. In these matings and transductional crosses, in which we wished to use $p d x L$ as an unselected marker, it was necessary to use a recipient strain in which the de novo synthetic pathway for POL-P had been blocked by a mutation such as $p d x B$ that also carried several selectable mutations in the region from 49 to $52 \mathrm{~min}$. For this purpose strain $\mathrm{AB} 2557 \mathrm{~F}$ ( $d s d A$ aro $C p d x B$ ) was infected with $\mathrm{P} 1$ grown on strain SN372L-3-9 and the transductants formed were first selected for the inheritance of both $d s d A^{+}$and $\operatorname{aro} C^{+}$ simultaneously on plates containing PAL, followed by scoring the number of those transductants which had also acquired $p d x L$. Among 45 transductants, only 2 required PAL for growth. This suggested that $p d x L$ is situated at about $1.0 \mathrm{~min}$ clockwise from aro $C$. This was further supported by an experiment in which transduction was performed using strain CAG18565 (nupC3146: : Tn10kan)

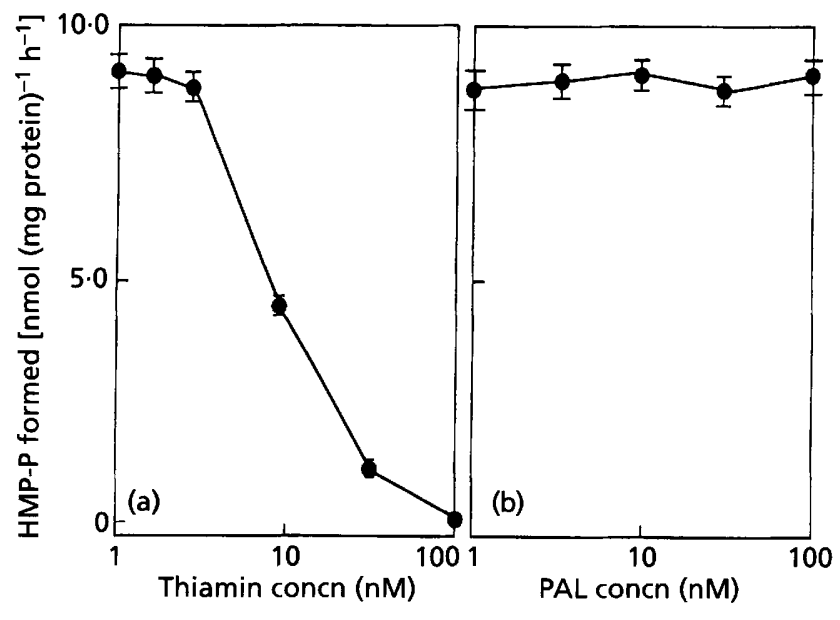

Fig. 2. Formation of HMP-P from HMP by extracts prepared from $E$. coli SN372L-3-9 cells grown in minimal media containing increasing concentrations of thiamin plus $100 \mathrm{nM}$ PAL (a) or increasing concentrations of PAL plus $3 \mathrm{nM}$ thiamin (b). The reactions and assay systems are described in Methods. Results shown are the means \pm SD of three different experiments.

as donor and strain SN372L-3-9 as recipient. In this cross, if $n u p C$ and $p d x L$ are located very close together, $\mathrm{Km}^{\mathrm{r}}$ $p d x L^{+} p d x B$ transductants would appear at high frequencies. The results showed that 36 of $42 \mathrm{Km}^{\mathrm{r}}$ transductants had gained the ability to grow on POL in the presence of HMP, whereas the remaining transductants still required PAL under the same conditions. On the other hand, when strain SN372L-3-9 was replaced by strain SN372L-3-9-10 in the same cross and the cotransduction of $\mathrm{Km}^{\mathrm{r}}$ with $p d x \mathrm{~L}^{+}$was examined, 23 of $27 \mathrm{Km}^{\mathrm{r}}$ transductants had gained the ability to grow on POL in the presence of thiamin. These results indicated that the $p d x L$ gene is situated very close to $n u p C$, probably within less than $0 \cdot 1 \mathrm{~min}$.

\section{Isolation and characterization of this mutant}

The tbiC (Ts) $p d x B$ (Ts) $p d x L$ mutant (SN372L-3-9) was mutagenized with NTG and cultured in minimal medium containing $1 \mathrm{mM}$ glycolaldehyde at $25^{\circ} \mathrm{C}$ for $40 \mathrm{~h}$. Methylthiohydroxymethylpyrimidine-resistant mutants were selected by a method essentially the same as that used in the selection for deoxypyridoxine resistance. In this case a paper disk which contained $0 \cdot 1 \mu \mathrm{mol}$ of this HMP analogue was placed on minimal agar plate supplemented with $1 \mathrm{mM}$ glycolaldehyde. Mutants that could grow on $20 \mathrm{nM}$ thiamin but not on the same concentration of HMP at $37^{\circ} \mathrm{C}$ were then selected on minimal medium containing glycolaldehyde. One such mutant, strain SN372L-3-9-10, was used for further studies.

Preliminary matings placed the site of the thiJ gene near $46 \mathrm{~min}$, presumably close to a region where two thiamin genes, thiD and $t h i M$, are located (Imamura \& Nakayama, 1981; Mizote \& Nakayama, 1989b). In this report, we determined cotransduction between thiJ and thiM with 
Table 2. Phosphorylation of HMP, POL and PAL by extracts from mutant cells grown at different concentrations of thiamin

Extracts prepared from cells grown on the indicated concentrations of thiamin plus $100 \mathrm{nM}$ PAL were incubated with each of the three substrates, HMP, POL and PAL in the reaction mixture. The amount of the corresponding phosphate ester formed was then measured. Results are given in nmol phosphate ester formed ( $m g$ protein) $)^{-1} \mathrm{~h}^{-1}$ and are the means $\pm \mathrm{sD}$ of three different experiments.

\begin{tabular}{|c|c|c|c|c|}
\hline \multirow{2}{*}{$\begin{array}{l}\text { Strain } \\
\text { (Relevant genotype)* }\end{array}$} & \multirow{2}{*}{ Phosphate ester formed } & \multicolumn{3}{|c|}{ Thiamin concn $(\mathrm{nm})$} \\
\hline & & 3 & 20 & 100 \\
\hline $\begin{array}{l}\text { SN372L-3 } \\
\left(p d x L^{+} t h i j^{+}\right)\end{array}$ & $\begin{array}{l}\text { HMP-P } \\
\text { POL-P } \\
\text { PAL-P }\end{array}$ & $\begin{array}{l}7 \cdot 82 \pm 0 \cdot 44 \\
1 \cdot 05 \pm 0 \cdot 06 \\
1 \cdot 87 \pm 0 \cdot 22\end{array}$ & $\begin{array}{l}8.94 \pm 0.29 \\
1.12 \pm 0.10 \\
1.35 \pm 0.13\end{array}$ & $\begin{array}{l}8.81 \pm 0.33 \\
1 \cdot 18 \pm 0.09 \\
1 \cdot 21 \pm 0.14\end{array}$ \\
\hline $\begin{array}{l}\text { SN372L-3-9 } \\
\left(p d x L \text { thi } J^{+}\right)\end{array}$ & $\begin{array}{l}\text { HMP-P } \\
\text { POL-P } \\
\text { PAL-P }\end{array}$ & $\begin{array}{l}6.00 \pm 0.33 \\
0.21 \pm 0.03 \\
0.97 \pm 0.09\end{array}$ & $\begin{array}{l}0.33 \pm 0.04 \\
0.20 \pm 0.04 \\
0.80 \pm 0.12\end{array}$ & $\begin{array}{c}<0.01 \\
<0.01 \\
1.01 \pm 0.11\end{array}$ \\
\hline $\begin{array}{l}\text { SN372L-3-9-10 } \\
(p d x L \text { thiJ) }\end{array}$ & $\begin{array}{l}\text { HMP-P } \\
\text { POL-P } \\
\text { PAL-P }\end{array}$ & $\begin{array}{c}<0.01 \\
<0.01 \\
0.17 \pm 0.04\end{array}$ & $\begin{array}{c}<0.01 \\
<0.01 \\
0.22 \pm 0.04\end{array}$ & $\begin{array}{c}<0.01 \\
<0.01 \\
0.19 \pm 0.04\end{array}$ \\
\hline
\end{tabular}

* Plus thiC $p d x B$ for all strains.

strain SN164L-1 (thi $A$ thiM) as donor and strain SN372L3-9-10 as recipient. Since in the recipient strain $t h i C, p d x B$ and $p d x L$ mutations are all further away from thiM than the maximal length of the chromosomal fragment carried by a single phage particle, three types of transductants with respect to the thiM mutation, thiJ thiM, thiJ thiM and $t b i J^{+} t h i M^{+}$, would appear in this cross. Transductants that had received thij ${ }^{+}$could be selected for their ability to grow on a combination of PAL and HMP. If the thiJ and thiM genes are situated close together, the unselected donor trait $t h i M$ would be cotransduced with $t b i j^{+}$at high frequencies. To determine whether or not the transductants had received the thiM mutation, HET kinase activity was assayed in cell extracts by the procedure described previously (Mizote \& Nakayama, 1989b). The results showed that 31 of 39 thi $^{+}$transductants possessed only about $1 / 20$ to $1 / 40$ the HET kinase activity of strain SN372L-3-9-10, while in the remaining 8 thij $^{+}$transductants the kinase activities were comparable to those of the recipient, showing that the thiJ mutation is $78-80 \%$ linked to the thiM allele.

\section{Phosphorylation of HMP, POL and PAL by extracts of mutant cells}

To investigate whether or not a regulatory mechanism is involved in the synthesis of the thiJ-encoded enzyme, mutant cells were grown in two different series of minimal media, one containing increasing concentrations of thiamin and $100 \mathrm{nM}$ PAL and the other containing increasing concentrations of PAL and $3 \mathrm{nM}$ of thiamin. The data obtained clearly showed that increasing the concentration of thiamin in the growth medium resulted in decreasing levels of HMP kinase activity (Fig. 2a). The concentration of thiamin that caused full repression of enzyme activity was above $100 \mathrm{nM}$, full derepression being evident at thiamin concentrations below $3 \mathrm{nM}$. Contrasting results were obtained with PAL; in this case the activity remained at a constant level at all concentrations (Fig. 2b).

Subsequently, the phosphorylation of HMP, POL and PAL were assayed with extracts from mutant cells grown in minimal medium supplemented with $100 \mathrm{nM}$ PAL together with thiamin at final concentrations of 3,20 and

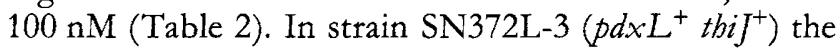
amount of the phosphate esters formed from the respective substrates appeared to be almost independent of the concentration of thiamin added to minimal medium, though the extracts showed the kinase activity for HMP to be much higher than either for POL or for PAL. In strain SN372L-3-9 ( $\left.p d x L_{\text {thi }}{ }^{+}\right)$neither HMP-P nor POL$\mathrm{P}$ was formed by extracts from cells grown on thiamin at $100 \mathrm{nM}$. Although decreasing the concentration of thiamin in the growth medium resulted in increased levels of HMP kinase activity, only a small amount of POL-P formation could be detected under the same conditions. With regard to the phosphorylation of PAL, the extracts showed approximately $60-80 \%$ as much activity as did extracts from strain SN372L-3. The addition of thiamin to the medium did not have any effect on the kinase activity toward this substrate. In strain SN372L-3-9-10 ( $p d x L$ thiJ), neither HMP-P nor POL-P was formed by extracts from cells grown on thiamin even at a concentration as low as $3 \mathrm{nM}$, at which thiamin limited the growth yield. Contrary to expectation, the formation of PAL-P could still be demonstrated, though the activity was only $20 \%$ of that of strain SN372L-3-9. The most reasonable explanation of these results is that two distinct enzymes are involved in the phosphorylation of HMP: an enzyme 
Table 3. Formation of HMP-P and HMP-PP from HMP by cell suspensions of mutant strains

Cells grown on $100 \mathrm{nM}$ PAL and $3 \mathrm{nM}$ thiamin were incubated with HMP in the reaction mixture. The amounts of HMP, HMP$\mathrm{P}$ and HMP-PP in the cells were then measured. Results are given in nmol HMP phosphate formed (mg dry wt cells) ${ }^{-1} \mathrm{~h}^{-1}$ and are the means $\pm S D$ of three different experiments.

\begin{tabular}{|lccc|}
\hline Strain & Relevant genotype* & HMP-P & HMP-PP \\
\hline SN372L-3 & $p d x L^{+}$thij & $1 \cdot 21 \pm 0 \cdot 10$ & $0.55 \pm 0.09$ \\
SN372L-3-9 & $p d \times L$ thij & $1 \cdot 05 \pm 0 \cdot 11$ & $0 \cdot 14 \pm 0.02$ \\
SN372L-3-9-10 & $p d x L$ thiJ & $<0.01$ & $<0.01$ \\
M3-9-6 & $p d x L$ thij & $3.33 \pm 0 \cdot 14$ & $8.75 \pm 0.34$ \\
K3-9-101 & $p d x L^{+}$thiJ & $0 \cdot 43 \pm 0.09$ & $<0.01$ \\
\hline
\end{tabular}

* Plus thiC $p d \times B$ for all strains.

previously designated pyridoxine kinase ( $\mathrm{PdxL}$ kinase) and an enzyme that has not previously been genetically analysed, now referred to as an HMP kinase (ThiJ kinase). The activity of the thij-encoded kinase can be measured only in strains with a defective pyridoxine kinase grown with a limited availability of thiamin. Under these conditions the expression of the thiJ gene is increased. The present results also suggest that, apart from the two kinases, no other enzymes catalyse the phosphorylation of HMP. The existence of a third enzyme that might catalyse the formation of PAL-P from PAL more efficiently than that of POL-P from POL is suggested since strain SN372L-3-9-10 was capable of catalysing the PAL phosphorylation reaction at an appreciable level and growth was still supported by thiamin plus PAL.

\section{Physiological effects of the this and the $p d x L$ mutations}

To obtain a more complete understanding of the physiological role of the $p d x L$ and thiJ genes in the biosynthesis of thiamin-PP, the $p d x L$ mutation of strain SN372L-3-910 was transduced into strain SN372L-3 and the transductants were tested for their ability to phosphorylate HMP. Extracts from 10 of the transductants (SN372L-3T1 to SN372L-3-T10) produced 6.20 $\pm 0.32 \mathrm{nmol} \mathrm{HMP-P}$ under the standard assay conditions when they were grown on a limited amount of thiamin, whereas no appreciable kinase activity could be detected when the transductants were grown in the presence of excess thiamin. The introduction of the $p d x L$ mutation into strain SN372L-3 therefore resulted in a mutant having the same characteristics as strain SN372L-3-9 with respect to the phosphorylation of HMP.

When $E$. coli cell suspensions were incubated under aerobic conditions with HMP in the presence of glucose, HMP was taken up into the cell and HMP-PP was formed in large amounts via the intermediate formation of HMP-P (Watanabe et al, 1977). The thiD gene is reported to encode HMP-P kinase which catalyses the reaction from HMP-P to HMP-PP (Imamura \& Nakayama, 1981). To determine the effect of the $p d x L$ and the thiJ mutations on the stepwise phosphorylation of HMP in intact cells, the relative rates of formation of HMP-P and HMP-PP from HMP were measured in a series of different strains including a $p d x L^{+}$thiJ transductant (Table 3). The results showed that in reaction mixtures containing cell suspensions of either strain SN372L-3 or strain SN372L-3-9, both HMP-P and HMP-PP were formed from HMP which had been taken up into the cell, whereas in strain SN372L-3-9-10 neither of the two phosphate esters were found, though the exogenously added HMP was detected within the cell. However, the inability of the latter strain to produce HMP-PP could not be considered to be direct evidence for the loss of HMP$P$ kinase, since it carries mutations in $p d x L$ and thiJ. Subsequently, two types of transductants that had acquired the wild-type allele corresponding to the $p d x L$ or thiJ mutation were constructed with strain SN372L-39-10 as recipient. Experiments with cell suspensions of two different types of transductants, M3-9-6 ( $p d x L$ thij $\left.{ }^{+}\right)$ and $\mathrm{K} 3-9-101$ ( $\left.p d x L^{+} t h i J\right)$, revealed that the ability to carry out the stepwise phosphorylation of HMP was restored by introducing the wild-type allele corresponding to the thiJ mutation. Introducing the wild-type allele $\left(p d x L^{+}\right)$resulted in the restoration of the ability to phosphorylate HMP into HMP-P, but not to form HMPPP from HMP-P (Table 3). Subsequently, we noted the thiC (Ts) mutation carried by strain SN372L-3-9-10 and found that the strain could grow on minimal medium containing PAL at $25^{\circ} \mathrm{C}$ at a rate comparable to that of strain SN372L. This indicates that the $t b i C^{+}$tbij mutant can synthesize thiamin-PP through the de novo synthetic pathway. It thus appears that free HMP is not involved in the de novo synthesis of thiamin-PP and that HMP-P might be the first pyrimidine compound synthesized endogenously. These results are consistent with those of Yamada \& Kumaoka (1982), who showed that in E. coli B the C5 and hydroxymethyl carbons of HMP are directly derived from a two-carbon fragment consisting of $4^{\prime}, 5^{\prime}$ carbon atoms of the phosphoribosyl moiety of 5 -aminoimidazole ribotide. The failure to isolate $p d x L$ mutants from strains prototrophic for POL also suggests that the phosphorylation of POL might not be involved in the de novo synthesis of PAL-P, and hence POL-P is probably the first pyridine compound arising in the pathway.

Genes involved in thiamin-PP biosynthesis have been shown to be located on the chromosome in four distinct regions. Mutations in five of the genes (thiCEFGH), all of them involved in the biosynthesis of the pyrimidine and thiazole moieties of thiamin, have been located at $90 \mathrm{~min}$ on the E. coli genetic map. Since these genes are tightly linked to each other and coordinately expressed, they are expected to constitute the thi operon (Horn $e t$ al. 1993). Therefore, the interaction of the thi operon with the thiD, $M$ and $J$ cluster, which are distant from each other on the chromosome, must be defined in order to understand the genetic control of thiamin-PP biosynthesis in $E$. coli $\mathrm{K}-12$. 


\section{ACKNOWLEDGEMENTS}

We thank Dr D. D. S. Smith, Institute of Food Research, Reading, UK, for his help with preparation of the manuscript.

\section{REFERENCES}

Adelberg, E. A., Mandel, M. \& Chen, G. C. (1965). Optimal conditions for mutagenesis by $\mathrm{N}$-methyl-N'-nitro-N-nitrosoguanidine in Eschericbia coli K-12. Biophys Biochem Res Commun 18, 788-795.

Bachmann, B. J. (1983). Linkage map of Escherichia coli K-12, 7th edn. Bacteriol Rev 47, 180-230.

Henderson, H. M. (1965). The conversion of pyridoxine phosphate into pyridoxal phosphate in Escherichia coli. Biochem J 95, 775-779.

Horn, P. B. V., Backstrom A. D., Stewart, V. \& Begley, T. P. (1993). Structural genes for thiamine biosynthetic enzymes $(t b i C E F G H)$ in Escherichia coli K-12. J Bacteriol 175, 982-992.

Imamura, N. \& Nakayama, H. (1981). thiD locus of Escherichia coli. Experientia 37, 1265-1266.

Imamura, N. \& Nakayama, H. (1982). thiK and thiL loci of Escherichia coli. J Bacteriol 151, 708-717.

Kawasaki, T., Nakata, T. \& Nose, Y. (1968). Genetic mapping with a thiamine-requiring auxotroph of Escherichia coli K-12 defective in thiamine phosphate pyrophosphorylase. J Bacteriol 95, 1483-1485.

Lam, H.-M. \& Winkler, M. E. (1992). Characterization of the complex $p d x H-t y r S$ operon of Escherichia coli K-12 and pleiotrophic phenotypes caused by $p d x H$ insertion mutations. $J$ Bacteriol 174, 6033-6045.

Lederberg, J. \& Lederberg, E. M. (1952). Replica plating and indirect selection of bacterial mutants. J Bacteriol 63, 399-406.

Lennox, E. S. (1955). Transduction of linked genetic characters of the host by bacteriophage P1. Virology 1, 190-206.

Lewin, L. M. \& Brown, G. M. (1961). The biosynthesis of thiamine. III. Mechanism of enzymatic formation of the pyrophosphate ester of 2-methyl-4-amino-5-hydroxymethylpyrimidine. J Biol Chem 236, 2768-2771.

Low, K. B. (1973). Rapid mapping of conditional and auxotrophic mutations in Eschericbia coli K-12 . J Bacteriol 113, 798-812.
Miller, J. H. (1972). Experiments in Molecular Genetics. Cold Spring Harbor, NY: Cold Spring Harbor Laboratory.

Mizote, T. \& Nakayama, H. (1989a). Purification and properties of hydroxymethylpyrimidine kinase from Escherichia coli. Biochim Biophys Acta 991, 109-113.

Mizote, T. \& Nakayama, H. (1989b). The thiM locus and its relation to phosphorylation of hydroxyethylthiazole in Escherichia coli. J Bacteriol 171, 3228-3232.

Nakayama, H. (1990). Genetic analysis of thiamin pyrophosphate biosynthesis in Escherichia coli. Vitamins 64, 619-632.

Nakayama, H. \& Hayashi, R. (1972a). Biosynthesis of thiamine pyrophosphate in Escherichia coli. J Bacteriol 109, 936-938.

Nakayama, H. \& Hayashi, R. (1972b). Biosynthetic pathway of thiamine pyrophosphate: a special reference to the thiamine monophosphate-requiring mutant and the thiamine pyrophosphate-requiring mutant of Escherichia coli. J Bacteriol 112, $1118-1126$

Nakayama, H. \& Hayashi, R. (1979). Isolation and characterization of Escherichia coli mutants auxotrophic for thiamine phosphates. Methods Ensymol 62, 94-101.

Osborn, M., Person, S., Philips, S. \& Funke, F. (1967). A determination of mutagen specificity in bacteria using nonsense mutants of bacteriophage T4. J Mol Biol 26, 437-447.

Shimizu, S. \& Dempsey, B. W. (1976). Genetic map position of the $p d x H$ gene in Escherichia coli. J Bacteriol 127, 1593-1594.

Taylor, A. L. \& Trotter, C. D. (1967). Revised linkage map of Escherichia coli. Bacteriol Rev 31, 332-353.

Turner, J. M. \& Happold, F. C. (1961). Pyridoxamine phosphateoxidase and pyridoxal phosphate-phosphatase activities in Escherichia coli. Biocbem J 78, 364-372.

Yamada, K. \& Kumaoka, H. (1982). Incorporation of a two-carbon fragment derived from ribose of 5-aminoimidazole ribotide into the pyrimidine moiety of thiamine. Biochem Int 5, 771-776.

Watanabe, K., Isoi, K., Nakayama H. \& Hayashi, R. (1977). Some properties of a mutant strain of Escherichia coli requiring high concentration of 2-methyl-4-amino-5-hydroxymethylpyrimidine. $J$ Nutr Sci Vitaminol 23, 81-93.

Received 26 March 1996; revised 25 June 1996; accepted 2 July 1996. 